\title{
Self-consistent tight-binding description of Dirac points moving and merging in two-dimensional optical lattices
}

\author{
Julen Ibañez-Azpiroz, ${ }^{1,2}$ Asier Eiguren, ${ }^{1,2}$ Aitor Bergara, ${ }^{1,2,3}$ Giulio Pettini, ${ }^{4}$ and Michele Modugno ${ }^{5,6}$ \\ ${ }^{1}$ Depto. de Física de la Materia Condensada, Universidad del Pais Vasco, UPV/EHU, 48080 Bilbao, Spain \\ ${ }^{2}$ Donostia International Physics Center (DIPC), 20018 Donostia, Spain \\ ${ }^{3}$ Centro de Física de Materiales CFM, Centro Mixto CSIC-UPV/EHU, 20018 Donostia, Spain \\ ${ }^{4}$ Dipartimento di Fisica e Astronomia, Università di Firenze, and INFN, 50019 Sesto Fiorentino, Italy \\ ${ }^{5}$ Depto. de Física Teórica e Hist. de la Ciencia, Universidad del Pais Vasco UPVIEHU, 48080 Bilbao, Spain \\ ${ }^{6}$ IKERBASQUE, Basque Foundation for Science, 48011 Bilbao, Spain
}

(Received 7 June 2013; published 30 September 2013)

\begin{abstract}
We present an accurate ab initio tight-binding model, capable of describing the dynamics of Dirac points in tunable honeycomb optical lattices following a recent experimental realization [Tarruell et al., Nature (London) 483, 302 (2012)]. Our scheme is based on first-principle maximally localized Wannier functions for composite bands. The tunneling coefficients are calculated for different lattice configurations, and the spectrum properties are well reproduced with high accuracy. In particular, we show which tight-binding description is needed in order to accurately reproduce the position of Dirac points and the dispersion law close to their merging, for different laser intensities.
\end{abstract}

DOI: 10.1103/PhysRevA.88.033631

PACS number(s): 67.85.Hj, 03.75.Lm

\section{INTRODUCTION}

The possibility to simulate graphenelike structures and investigate the physics of Dirac points with ultracold atoms in optical lattices is attracting an increasing interest in the literature [1-11]. Recently, Tarruell et al. [8] have reported the creation and manipulation of Dirac points in a tunable honeycomb optical lattice, exploring the topological transition taking place at the merging of Dirac points, and comparing their experimental results with ab initio calculations of the Bloch spectrum. The same experiment has also been interpreted in Ref. [9] by means of a tight-binding model defined on a square lattice and of an universal tight-binding Hamiltonian that provides a low-energy effective description in the vicinity of the merging of Dirac points, describing the corresponding topological transition between a semimetallic phase and a band insulator [5]. Though this model remarkably captures all the relevant physics, its connection with the optical lattice parameters is indirect and has some limitations, as it relies on a fit of the parameters of the universal Hamiltonian to the two lowest-lying energy bands in the vicinity of the Dirac cones $[9,11]$.

In this paper, we present a comprehensive scheme based on composite maximally localized Wannier functions (MLWFs) [12] for constructing an ab initio tight-binding model corresponding to the tunable honeycomb potential with two minima per unit cell as described in Ref. [8]. The MLWFs are obtained by means of a gauge transformation that minimizes their spread, a procedure that is routinely employed in condensedmatter physics [13]. As it has been recently demonstrated, these functions represent an optimal tool for constructing tight-binding models for ultracold atoms in optical lattices [14-16], allowing for an accurate mapping of the system Hamiltonian onto a discrete model defined on a lattice. In particular, MLWFs provide a well-defined way for the ab initio calculation of the relevant tight-binding parameters, with a fine control over next to leading corrections.
The paper is organized as follows. In Sec. II, we review the general approach for mapping a continuous many-body Hamiltonian onto a discrete tight-binding model by means of MLWFs. In Sec. III we apply this scheme to the tunable two-dimensional honeycomb lattice of Ref. [8], discussing the general structure of the associated Bravais lattice in direct and reciprocal space, and presenting the tight-binding expansion up to the third-nearest neighbors. Additionally, we provide explicit numerical results for the calculated MLWFs, the inferred tunneling coefficients, and the Bloch band structure. Then, in Sec. IV, we make use of the tight-binding Hamiltonian in reciprocal space-expressed in terms of tunneling coefficients and functions depending on the geometry of the associated lattice-for discussing the behavior of the Dirac points as a function of the lattice parameters. In this section, we also analyze the dispersion relation close to the merging of Dirac points, refining and improving the analysis of Refs. [5,9]. In addition, we discuss the effect of parity breaking which destroys the degeneracy of the two potential minima and provides a finite Dirac mass. Some accessory but important details are included in the Appendixes, covering the tight-binding expansion, the gauge dependence of the results, and the numerical application.

\section{TIGHT-BINDING EXPANSION AND MLWFs}

Let us consider a many-body system of bosonic or fermionic particles described by the field operator $\hat{\psi}(\boldsymbol{r})$. Since the physics of Dirac points is determined by the single-particle spectrum, we consider the following noninteracting manybody Hamiltonian,

$$
\hat{\mathcal{H}}_{0}=\int d \boldsymbol{r} \hat{\psi}^{\dagger}(\boldsymbol{r}) \hat{H}_{0} \hat{\psi}(\boldsymbol{r}),
$$

with $\hat{H}_{0}=-\left(\hbar^{2} / 2 m\right) \nabla^{2}+V(\boldsymbol{r})$ and the lattice periodic potential $V(\boldsymbol{r})=V(\boldsymbol{r}+\boldsymbol{R})$, where $\boldsymbol{R}$ belongs to the associated 
Bravais lattice. We remark that the optimal Wannier basis is solely determined by the single-particle spectrum, even though the inclusion of an interaction would be straightforward $[14,16,17]$ and the derivations following the next lines would not be affected.

The Hamiltonian (1) can be conveniently mapped onto a discrete lattice corresponding to the minima of the potential $V(\boldsymbol{r})$ by expanding the field operator in terms of a set of functions $\left\{w_{j v}(\boldsymbol{r})\right\}$ localized at each minimum,

$$
\hat{\psi}(\boldsymbol{r}) \equiv \sum_{j v} \hat{a}_{\boldsymbol{j} v} w_{j v}(\boldsymbol{r})
$$

where $v$ is a band index and $\hat{a}_{j v}^{\dagger}\left(\hat{a}_{j v}\right)$ represent the creation (destruction) operators of a single particle in the $j$ th cell. These operators satisfy the usual commutation (or anticommutation) rules following from those of the field $\hat{\psi}$.

The MLWFs, introduced by Marzari and Vanderbilt in [12], are obtained through a unitary transformation of the Bloch eigenstates,

$$
\left\langle x \mid w_{\boldsymbol{j} v}\right\rangle=\frac{1}{\sqrt{V_{\mathcal{B}}}} \int_{\mathcal{B}} d \boldsymbol{k} e^{-i \boldsymbol{k} \cdot \boldsymbol{R}_{j}} \sum_{m=1}^{N} U_{v m}(\boldsymbol{k})\left\langle x \mid \psi_{m \boldsymbol{k}}\right\rangle,
$$

with $V_{\mathcal{B}}$ the volume of the first Brillouin zone and $U \in U(N)$ a gauge transformation which obeys periodicity conditions in order to preserve the Bloch theorem. This gauge transformation is obtained through the minimization of the localization functional $\Omega=\sum_{v}\left[\left\langle\boldsymbol{r}^{2}\right\rangle_{v}-\langle\boldsymbol{r}\rangle_{v}^{2}\right]$ [12]. The resulting MLWFs have the desirable property of being exponentially localized in coordinate space [18,19], constituting an ideal basis for tight-binding models [15]. In this article we consider the MLWFs for composite bands $(N>1)$ since we are interested in geometries where the Wigner-Seitz cell has a nontrivial basis. This allows each MLWF to be centered on a single potential minimum inside the elementary cell, in contrast to single-band Wannier functions $[14,15,20]$. For this work, the MLWFs have been computed by means of the WANNIER90 code $[13,21]$ and a modified version of the QUANTUM-ESPRESSO package [22] adapted to the case of an optical lattice [15]. We also mention that other methods for specific cases have been recently proposed $[14,16]$.

The Hamiltonian (1) can be written in terms of Wannier states $\left|w_{j v}\right\rangle$ as

$$
\hat{\mathcal{H}}_{0}=\sum_{\nu \nu^{\prime}=A, B} \sum_{j, j^{\prime}} \hat{a}_{\boldsymbol{j} \nu}^{\dagger} \hat{a}_{\boldsymbol{j}^{\prime} v^{\prime}}\left\langle w_{\boldsymbol{j} v}\left|\hat{H}_{0}\right| w_{\boldsymbol{j}^{\prime} v^{\prime}}\right\rangle,
$$

where the matrix elements $\left\langle w_{j v}\left|\hat{H}_{0}\right| w_{j^{\prime} v^{\prime}}\right\rangle$ depend only on $\boldsymbol{i}=\boldsymbol{j}^{\prime}-\boldsymbol{j}$ due to the translational invariance of the lattice. These matrix elements correspond to tunneling amplitudes between different lattice sites, except for the special case $\boldsymbol{i}=\mathbf{0}$, $v=v^{\prime}$ that corresponds to on-site energies. Then, by defining

$$
\hat{d}_{\nu \boldsymbol{k}}=\frac{1}{\sqrt{V_{B}}} \sum_{j} e^{-i \boldsymbol{k} \cdot \boldsymbol{R}_{j}} \hat{a}_{\boldsymbol{j} \nu}
$$

$\hat{\mathcal{H}}_{0}$ is transformed as

$$
\hat{\mathcal{H}}_{0}=\sum_{\nu v^{\prime}} \int_{\mathcal{B}} d^{2} \boldsymbol{k} h_{\nu v^{\prime}}(\boldsymbol{k}) \hat{d}_{\nu \boldsymbol{k}}^{\dagger} \hat{d}_{v^{\prime} \boldsymbol{k}}
$$

with

$$
h_{\nu v^{\prime}}(\boldsymbol{k})=\sum_{i} e^{i \boldsymbol{k} \cdot \boldsymbol{R}_{i}}\left\langle w_{\mathbf{0} v}\left|\hat{H}_{0}\right| w_{\boldsymbol{i} v^{\prime}}\right\rangle
$$

being the Hamiltonian density in quasimomentum space, whose eigenvalues are in principle equal to the exact energy bands $\varepsilon_{v}(\boldsymbol{k})$. For practical purposes, however, the expression (7) must be truncated by retaining only a finite number of matrix elements. This corresponds to the tight-binding expansion in $\boldsymbol{k}$ space. The actual number of terms needed to reproduce the energy bands (or any other physical quantity) within a certain degree of accuracy crucially depends on the properties of the basis functions $w_{j v}(\boldsymbol{x})$. In this context, the MLWFs represent an optimal choice due to their minimal spread, as it is analyzed in detail in Appendix A.

In this paper will apply the above tight-binding expansion to the tunable two-dimensional honeycomb potential of the experiment [8].

\section{TUNABLE HONEYCOMB LATTICE}

The functional form of the potential reproduced experimentally in Ref. [8] is

$$
\begin{aligned}
V(x, y)= & -V_{\bar{X}} \cos ^{2}\left(k_{L} x+\theta / 2\right) \\
& -V_{X} \cos ^{2}\left(k_{L} x\right)-V_{Y} \cos ^{2}\left(k_{L} y\right) \\
& -2 \alpha \sqrt{V_{X} V_{Y}} \cos \left(k_{L} x\right) \cos \left(k_{L} y\right) \cos (\varphi),
\end{aligned}
$$

where all the parameters can be controlled and tuned in the experiment. In particular, by varying the laser intensities $V_{\bar{X}}, V_{X}$, and $V_{Y}$, several structures can be realized by continuous deformations, including square, triangular, checkerboard, dimer, honeycomb, and one-dimensional (1D) chain geometries [8]. Let us define the Bravais lattice associated to the potential minima as $\mathcal{B}=\left\{\boldsymbol{R}_{m n}=m \boldsymbol{a}_{1}+n \boldsymbol{a}_{2} \mid m, n \in \mathbb{Z}\right\}$ (see Fig. 1), which is generated by the two basis vectors

$$
\boldsymbol{a}_{1,2}=\frac{\pi}{k_{L}}\left(\boldsymbol{e}_{x} \mp \boldsymbol{e}_{y}\right)
$$

with $\boldsymbol{e}_{x, y}$ being the Cartesian unit vectors. The associated reciprocal space basis vectors are

$$
\boldsymbol{b}_{1,2}=k_{L}\left(\boldsymbol{e}_{x} \mp \boldsymbol{e}_{y}\right)
$$

following from $\boldsymbol{a}_{i} \cdot \boldsymbol{b}_{j}=2 \pi \delta_{i j}$. From now on, we fix $k_{L}=$ $1, \hbar=1$, and $m=1 / 2$ without loss of generality. This corresponds to measuring lengths in units of $1 / k_{L}$ and energies in units of the recoil energy $E_{R}=\hbar^{2} k_{L}^{2} / 2 m$ [15].

Considering that the unit cell analyzed in this work contains two basis points ( $A$ and $B$ ), we will accordingly incorporate the contribution of the two lowest-energy bands to our formalism. It is then customary to write the Hamiltonian density in Eq. (7) as

$$
h(\boldsymbol{k})=\left(\begin{array}{cc}
\epsilon_{A}(\boldsymbol{k}) & z(\boldsymbol{k}) \\
z^{*}(\boldsymbol{k}) & \epsilon_{B}(\boldsymbol{k})
\end{array}\right) .
$$

Above, the band index $v=1,2$ [see Eq. (2)] has been traded to $v=A, B$ since the associated MLWFs are located around the minima $A$ and $B$. The two lowest-energy bands are then 


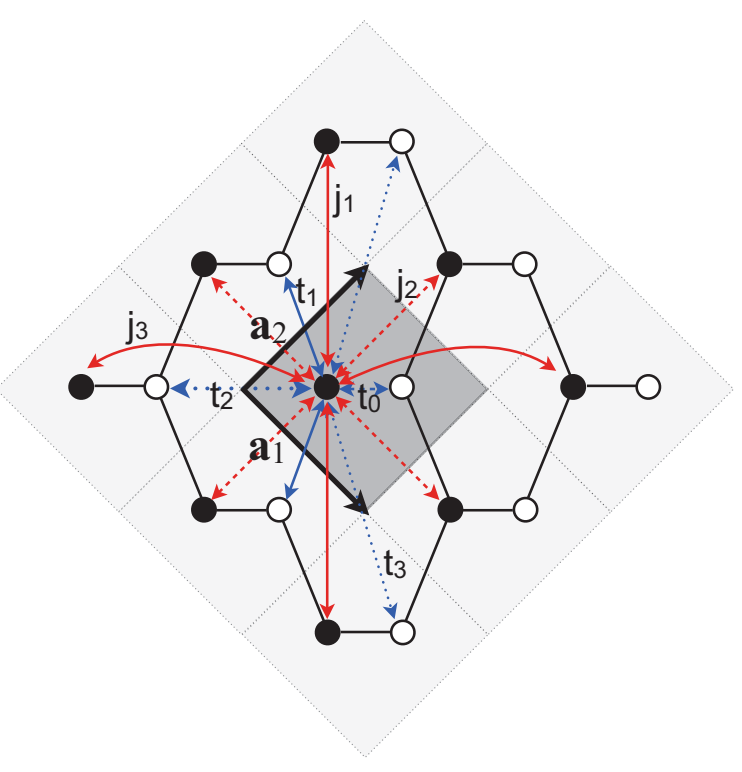

FIG. 1. (Color online) Bravais lattice associated to the potential in Eq. (8) for the stretched-honeycomb configuration. Black and white circles refers to minima of type $A$ and $B$, respectively. The elementary cell is highlighted in gray. The various diagonal and off-diagonal tunneling coefficients of our tight-binding expansion are indicated for the site of type $A$ in the central cell.

given by the eigenvalues of (11),

$$
\varepsilon_{ \pm}(\boldsymbol{k})=\epsilon_{+}(\boldsymbol{k}) \pm \sqrt{\epsilon_{-}^{2}(\boldsymbol{k})+|z(\boldsymbol{k})|^{2}}
$$

with $\epsilon_{ \pm}(\boldsymbol{k})=\left[\epsilon_{A}(\boldsymbol{k}) \pm \epsilon_{B}(\boldsymbol{k})\right] / 2$.

The matrix elements in (11) can be expanded as

$$
\begin{aligned}
& \epsilon_{\nu}(\boldsymbol{k})=\sum_{m n} J_{m n}^{v} e^{-i \boldsymbol{k} \cdot \boldsymbol{R}_{m n}}, \\
& z(\boldsymbol{k})=-\sum_{m n} T_{m n} e^{-i \boldsymbol{k} \cdot \boldsymbol{R}_{m n}},
\end{aligned}
$$

with

$$
\begin{gathered}
J_{m n}^{v} \equiv\left\langle w_{v}^{\mathbf{0}}\left|\hat{H}_{0}\right| w_{v}^{\boldsymbol{R}_{m n}}\right\rangle, \\
T_{m n} \equiv-\left\langle w_{A}^{\mathbf{0}}\left|\hat{H}_{0}\right| w_{B}^{\boldsymbol{R}_{m n}}\right\rangle
\end{gathered}
$$

corresponding to diagonal and off-diagonal matrix elements, respectively. The sign convention is chosen in such a way that all the coefficients are positive defined [15]. In our model, we truncate the tight-binding expansion in order to include all possible tunneling coefficients between neighboring cells, as indicated in Fig. 1 [23]. This expansion is equivalent to that of Ref. [10].

Let us start by considering the diagonal terms. We fix an arbitrary energy offset to obtain

$$
\begin{gathered}
\epsilon_{A}(\boldsymbol{k})=\epsilon+F^{A}(\boldsymbol{k}), \\
\epsilon_{B}(\boldsymbol{k})=-\epsilon+F^{B}(\boldsymbol{k}),
\end{gathered}
$$

with

$$
\epsilon=\left(J_{00}^{A}-J_{00}^{B}\right) / 2
$$

and

$$
\begin{aligned}
F^{\nu}(\boldsymbol{k})= & 2 j_{1}^{\nu} \cos \left(2 \pi k_{y}\right)+4 j_{2}^{\nu} \cos \left(\pi k_{y}\right) \cos \left(\pi k_{x}\right) \\
& +2 j_{3}^{v} \cos \left(2 \pi k_{x}\right) .
\end{aligned}
$$

The tunneling coefficients appearing in Eq. (20) connect the minima located at points of the same type ( $A$ or $B$; see Fig. 1), and have been redefined as follows:

$$
\begin{aligned}
& j_{1}^{\nu} \equiv J_{1-1}^{\nu}=J_{-11}^{v}, \\
& j_{2}^{\nu} \equiv J_{10}^{\nu}=J_{01}^{\nu}=J_{0-1}^{v}=J_{-10}^{v}, \\
& j_{3}^{v} \equiv J_{11}^{v}=J_{-1-1}^{v},
\end{aligned}
$$

in order to simplify the notations.

We notice that, when $\theta=\pi$, the potential minima located at $A$ and $B$ are degenerate in energy. As a consequence, we have that $\epsilon=0$ and $j_{i}^{A}=j_{i}^{B} \equiv j_{i}$, so that $F^{A}(\boldsymbol{k})=F^{B}(\boldsymbol{k}) \equiv F(\boldsymbol{k})$ and the eigenvalues in Eq. (12) are simplified to

$$
\varepsilon_{ \pm}(\boldsymbol{k})=F(\boldsymbol{k}) \pm|z(\boldsymbol{k})| .
$$

Regarding the off-diagonal matrix element $z(\boldsymbol{k})$, its analytical form is given by

$$
\begin{aligned}
z(\boldsymbol{k}) \equiv & -\left[t_{0}+2 t_{1} \cos \left(\pi k_{y}\right) e^{-i \pi k_{x}}+t_{2} e^{-2 i \pi k_{x}}\right. \\
& \left.+2 t_{3} \cos \left(2 \pi k_{y}\right)\right],
\end{aligned}
$$

where the tunneling coefficients have been redefined as

$$
\begin{aligned}
& t_{0} \equiv T_{00}, \quad t_{1} \equiv T_{10}=T_{01}, \\
& t_{2} \equiv T_{-1-1}, \quad t_{3} \equiv T_{1-1}=T_{-11} .
\end{aligned}
$$

Notice that the ordering of the tunneling coefficients in Eqs. (24) and (21) does not necessarily correspond to the hierarchy of their magnitudes, which depends on the regime of the potential parameters considered (see later on).

In the following, we will apply the above presented model to the stretched honeycomb configuration [24] and analyze the properties of the Dirac points. We will also discuss the effect of increasing the overall potential intensity as well as the effect of lifting the degeneracy associated to the sites $A$ and $B$.

\section{MLWFs AND TUNNELING COEFFICIENTS FOR THE DEGENERATE CASE}

In this section we will discuss the numerical results for the stretched-honeycomb configuration with two degenerate minima per unit cell, obtained by fixing $\theta=\pi, \varphi=0$ in (8). This is the most interesting configuration as it is characterized by the presence of massless Dirac points. The effect of parity breaking $(\theta \neq \pi)$, which generates a Dirac mass, will be analyzed in Sec. V B. In addition, in Appendix B we will present the results for a wider range of lattice configurations.

We start by considering the experimental regime of Tarruell et al. [8], namely $V_{X}=0.28, V_{Y}=1.8$, and $V_{\bar{X}}$ variable ranging from 3 to 6 . Within this range of parameters, the potential (8) has the stretched-honeycomb structure shown in Fig. 2(a). This configuration determines the shape of the calculated MLWFs, drawn in Fig. 2(b) for the sublattice of type $A$. As shown in this figure, the MLWF is exponentially localized around the $A$ site of the central unit cell (note the logarithmic scale), presenting a non-negligible contribution 

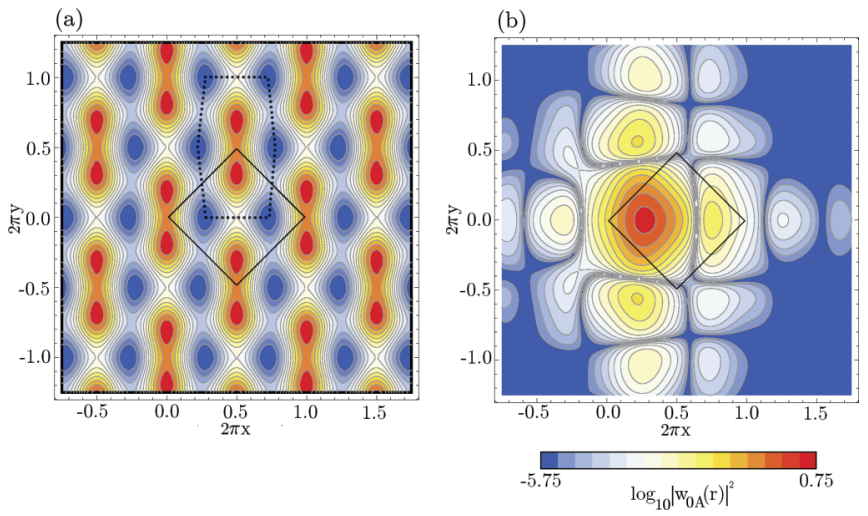

FIG. 2. (Color online) (a) Stretched-honeycomb potential (8) for $V_{\bar{X}}=5, V_{X}=0.28$, and $V_{Y}=1.8$. Hot and cold colors denote high and low values of the potential, respectively. The unit cell is indicated by solid (black) lines. (b) Structure of the calculated MLWF for sublattice $A$ for the same potential setup as in (a) (see text).

around the neighboring potential minima as well. The associated tunneling coefficients are illustrated in Fig. 3(a). This figure shows the behavior of the diagonal and off-diagonal coefficients, $t_{i}(i=0,3)$ and $j_{i}(i=1,3)$ as a function of $V_{\bar{X}}$.
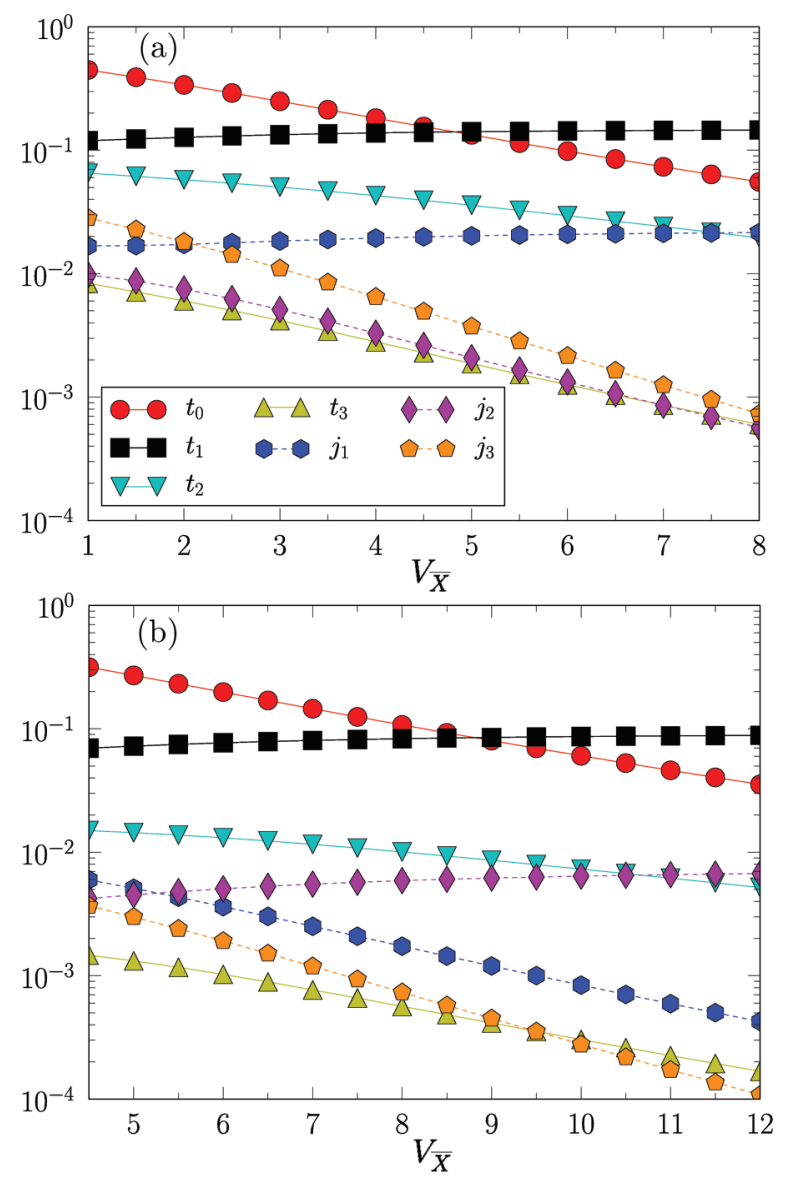

FIG. 3. (Color online) Behavior of the various tunneling coefficients as function of $V_{\bar{X}}$. Panel (a) covers the experimental regime, $V_{X}=0.28, V_{Y}=1.8$, while in (b) we consider a proper tight-binding regime, $V_{X}=0.56, V_{Y}=3.6$.
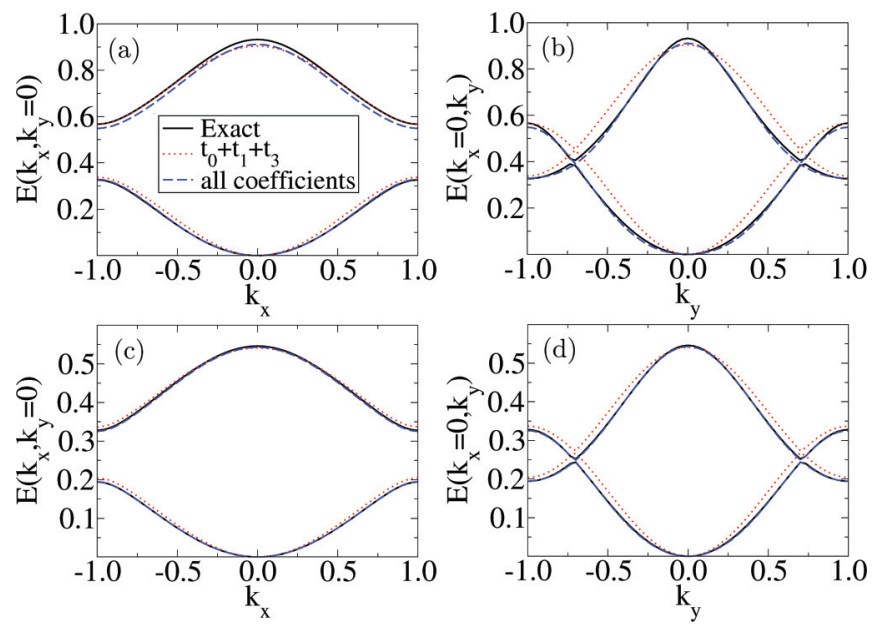

FIG. 4. (Color online) Cut of the exact energy bands (black solid line) compared to the two tight-binding approximations with just $t_{0}$, $t_{1}$, and $t_{2}$ (red dotted line), and with all the coefficients in Fig. 1 (green dotted-dashed line). Panels (a) and (b) respectively show cuts along $k_{x}\left(k_{y}=0\right)$ and $k_{y}\left(k_{x}=0\right)$, for $V_{X}=0.28, V_{Y}=1.8$, and $V_{\bar{X}}=5$; (c) and (d) refer to $V_{X}=0.56, V_{Y}=3.6$, and $V_{\bar{X}}=8.5$.

In Fig. 4 we present the tight-binding energy dispersion of Eq. (22) computed with the aid of the calculated tunneling coefficients. In particular, we consider two different tightbinding approximations: one includes just $t_{0}, t_{1}$, and $t_{2}$ (this corresponds to the universal Hamiltonian of Ref. [5]), while the other approximation includes all the coefficients defined in Fig. 1. In Figs. 4(a) and 4(b) we compare the energy dispersion of the different tight-binding approximations with the exact spectrum at $V_{\bar{X}}=5$. The figures show that the main features, such as the band crossing along the $k_{y}$ direction ([Fig. 4(b)], are well reproduced by both approximations. Quantitatively, however, the tight-binding model with just $t_{0}, t_{1}$, and $t_{2}$ is not capable of approximating the exact bands with sufficient accuracy (this holds in all the range of $V_{\bar{X}}$ considered in this paper).

At this point, we consider a different set of values for the potential parameters that correspond to a well-defined tightbinding regime, while maintaining the stretched-honeycomb structure. In particular, we use the parameter values $V_{X}=$ $0.56, V_{Y}=3.6$, and $V_{\bar{X}}$ ranging from 6 to 12 , corresponding to twice the values of Tarruell et al. [8]. The calculated tunneling coefficients are illustrated in Fig. 3(b), showing the same general structure as the ones in Fig. 3(a), except for minor differences regarding the smallest coefficients. The corresponding energy dispersion is shown in Figs. 4(c) and 4 (d) for $V_{\bar{X}}=8.5$. For this parameter configuration, even the lowest-order approximation with just the coefficients $t_{0}, t_{1}$, and $t_{2}$ provides a remarkable agreement with the exact data. This is also the case for the dimer and 1D-chain limits, as shown in Appendix C.

\section{DIRAC POINTS}

As we have seen in the previous section, the spectrum for a stretched-honeycomb configuration with $\theta=\pi$ is characterized by points where the two bands are degenerate, with a linear dispersion along at least one direction-the so-called 
Dirac points. These points are defined by $z\left(\boldsymbol{k}_{D}\right)=0$ and come always in pairs due to time-reversal invariance, which implies $z^{*}\left(\boldsymbol{k}_{D}\right)=z\left(-\boldsymbol{k}_{D}\right)[5,10]$. The existence and position of the Dirac points depends on the geometry of the lattice: in the case of a regular honeycomb structure, these points are located at the corners of the Brillouin zone $[4,15]$, whereas in the present tunable case the Dirac points can be moved inside the Brillouin zone, as shown in [8]. In particular, from the expression in Eq. (23), the position $\boldsymbol{k}_{D}=\left(k_{x}, k_{y}\right)$ of the Dirac points can be obtained by solving the following equation:

$$
t_{0}+2 t_{1} \cos \left(\pi k_{y}\right) e^{-i \pi k_{x}}+t_{2} e^{-2 i \pi k_{x}}+2 t_{3} \cos \left(2 \pi k_{y}\right)=0,
$$

whose imaginary part yields

$$
k_{x}=0
$$

inside the first Brillouin zone. Then, Eq. (25) becomes

$$
t_{0}+2 t_{1} \cos \left(\pi k_{y}\right)+t_{2}+2 t_{3} \cos \left(2 \pi k_{y}\right)=0 .
$$

Taking into account the hierarchy of the tunneling coefficients indicated in Fig. 3, the above equation is solved by

$$
k_{y}= \pm \frac{1}{\pi} \cos ^{-1}\left[\frac{-t_{1}+\sqrt{t_{1}^{2}+4 t_{3}\left(2 t_{3}-t_{0}-t_{2}\right)}}{4 t_{3}}\right] .
$$

In the regimes analyzed in this paper, this expression can be further approximated as

$$
k_{y} \simeq \pm \frac{1}{\pi} \cos ^{-1}\left[-\frac{t_{0}+t_{2}}{2 t_{1}}\right],
$$

which corresponds to the expression of Ref. [5]. Both Eq. (28) and its approximate version, Eq. (29), provide a valid solution when $t_{0}+t_{2} \leqslant 2 t_{1}$, which is satisfied also in the range of parameters corresponding to the stretched honeycomb, as shown in Fig. 5.
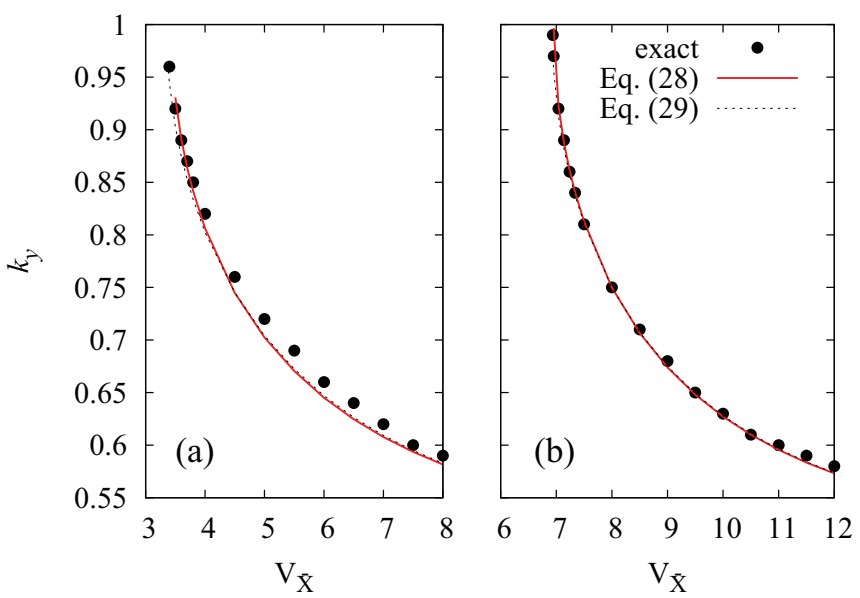

FIG. 5. (Color online) Position of the Dirac points along the $k_{y}$ axis as a function of $V_{\bar{X}}$ for (a) the parameter regime of Tarruell et al. [8], and (b) the tight-binding regime discussed in the text. The exact positions (circles) extracted from the Bloch spectrum are compared with the predictions of Eqs. (28) and (29).

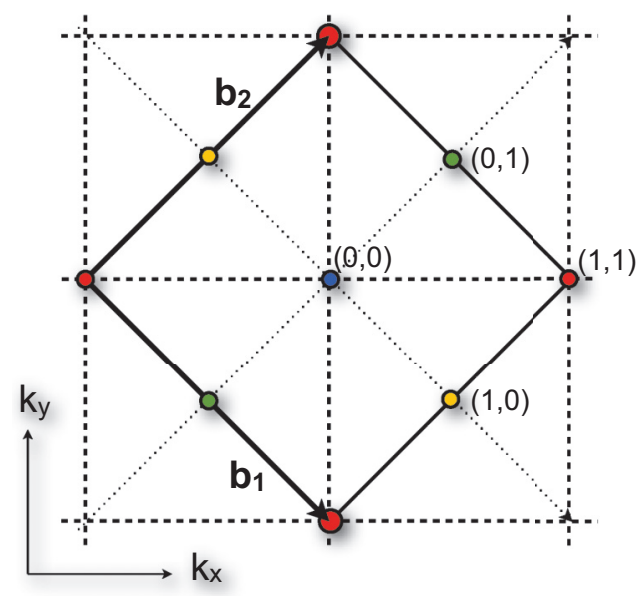

FIG. 6. (Color online) Unit cell in quasimomentum space, with the location of the possible merging of the Dirac points. Equivalent points (connected by a reciprocal space vector $\boldsymbol{G}$ ) are depicted with the same color. Given the actual values of the tunneling coefficients, only the points at $k_{x}=0, k_{y}= \pm 1$ can be realized (larger red dots).

\section{A. Merging of Dirac points}

The merging of Dirac points occurs when the two solutions of Eq. (28) coincide modulo a reciprocal space vector $\boldsymbol{G}=$ $p \boldsymbol{b}_{1}+q \boldsymbol{b}_{2}$ [with $p, q \in \mathbb{Z}$; see Eq. (9)], namely at $\boldsymbol{k}_{M}=$ $-\boldsymbol{k}_{M}+\boldsymbol{G}$. Therefore, the merging point $\boldsymbol{k}_{M}$ satisfies [5]

$$
\boldsymbol{k}_{M}=\boldsymbol{G} / 2=\frac{p \boldsymbol{b}_{\mathbf{1}}+q \boldsymbol{b}_{2}}{2} .
$$

In principle, there are four possible inequivalent merging points due to the geometry of the lattice, namely $(p, q)=$ $(0,0),(0,1),(1,0),(1,1)[5]$, which are illustrated in Fig. 6. However, considering the values of the tunneling coefficients, only the point $(1,1)$ and its equivalents are possible. In particular, the Dirac points inside the first Brillouin zone merge with those Dirac points located in the outer cells at the top and bottom corners $(1,-1)$ and $(-1,1)$, namely for $\boldsymbol{k}_{M} \equiv(0, \pm 1)$. For the two parameter regimes analyzed in this paper, the merging occurs at $V_{\bar{X}} \simeq 3.4$ (see also [8]) and $V_{\bar{X}} \simeq 6.94$, as respectively illustrated in Figs. 5(a) and 5(b).

Following Refs. [5,9], we now expand the Hamiltonian density around one of the two merging points by defining $\tilde{\boldsymbol{k}} \equiv \boldsymbol{k}-\boldsymbol{k}_{M}$. As discussed in Ref. [9], the general form of the off-diagonal component $z(\boldsymbol{k})$ around a merging point is characterized by a linear term in $\tilde{k}_{x}$ and a quadratic one in $\tilde{k}_{y}$, coming respectively from the imaginary and real parts of $z(\tilde{\boldsymbol{k}})$. The leading terms of the expansion are

$$
\begin{aligned}
& z_{R}(\tilde{\boldsymbol{k}}) \simeq-\left[t_{0}-2 t_{1}+t_{2}+2 t_{3}\right]+\pi^{2}\left[\left(4 t_{3}-t_{1}\right) \tilde{k}_{y}^{2}\right], \\
& z_{I}(\tilde{\boldsymbol{k}}) \simeq 2 \pi\left(t_{2}-t_{1}\right) \tilde{k}_{x} .
\end{aligned}
$$

In this paper we take into account the diagonal term $F(\boldsymbol{k})$, which was not included in the approach considered in Refs. [5,9]. This term affects the quadratic behavior and introduces an asymmetry between the two bands. Neglecting an unimportant constant term, we obtain the following expression:

$$
F(\tilde{\boldsymbol{k}}) \simeq-2 \pi^{2}\left(2 j_{1}-j_{2}\right) \tilde{k}_{y}^{2} .
$$



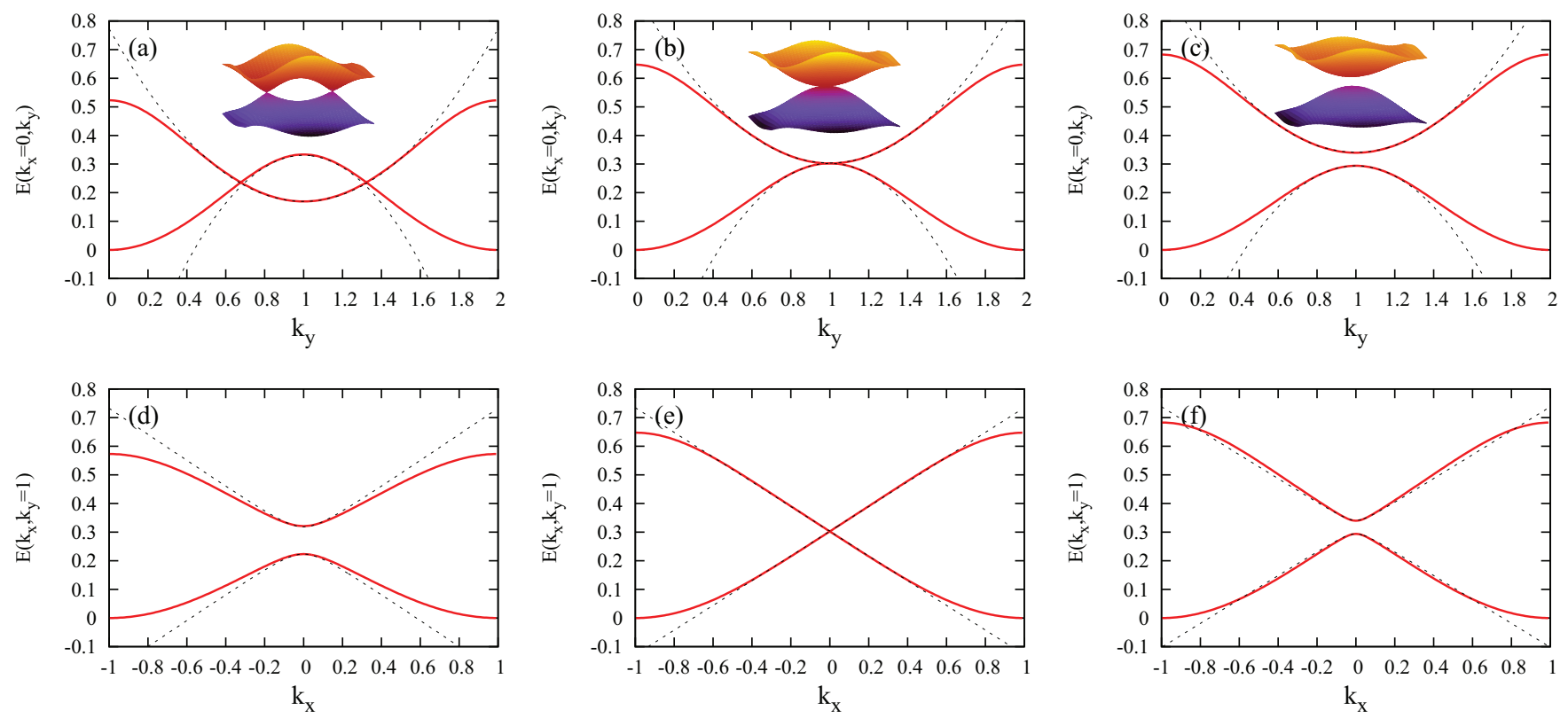

FIG. 7. (Color online) Cuts of the energy bands around the merging point $\boldsymbol{k}_{M}=(0,1)$, for the tight-binding regime discussed in the text ( $V_{X}=0.56, V_{Y}=3.6$ ). The exact Bloch bands (red solid lines) are compared to the approximate expressions in Eq. (38), as a function of $k_{y}$ (at $\left.k_{x}=0\right)$ [(a),(b),(c)], and of $k_{x}$ (at $\left.k_{y}=1\right)$ [(d),(e),(f)]. Each column corresponds to a different value of $V_{\bar{X}}$ : (a),(d) $V_{\bar{X}}=8$, (b),(e) $V_{\bar{X}}=6.94$ (merging point), and (c),(f) $V_{\bar{X}}=6.54$. Note that the cut along $k_{x}$ in (d) does not cross the Dirac point, as the latter is located at $k_{y} \simeq 0.68$; this is the reason why Eq. (38) provides a poorer approximation in this case.

Therefore, close to the merging point the Hamiltonian density can be cast into the form

$$
h_{v v^{\prime}}(\tilde{\boldsymbol{k}}) \simeq \frac{\tilde{k}_{y}^{2}}{2 \mu} \otimes I+\left(\Delta+\frac{\tilde{k}_{y}^{2}}{2 m^{*}}\right) \otimes \sigma_{x}+c \tilde{k}_{x} \otimes \sigma_{y}
$$

with

$$
\begin{gathered}
\Delta \equiv-\left[t_{0}-2 t_{1}+t_{2}+2 t_{3}\right], \\
\frac{1}{2 m^{*}} \equiv \pi^{2}\left(4 t_{3}-t_{1}\right), \\
c \equiv 2 \pi\left(t_{1}-t_{2}\right), \\
\frac{1}{2 \mu} \equiv-2 \pi^{2}\left(2 j_{1}-j_{2}\right) .
\end{gathered}
$$

The corresponding dispersion law is

$$
\varepsilon_{ \pm}(\tilde{\boldsymbol{k}}) \simeq \frac{\tilde{k}_{y}^{2}}{2 \mu} \pm \sqrt{\left(\Delta+\frac{\tilde{k}_{y}^{2}}{2 m^{*}}\right)^{2}+c^{2} \tilde{k}_{x}^{2}} .
$$

The parameter $\Delta$ vanishes at the merging point and marks the topological transition between semimetallic and insulating phases, which is driven by a change of sign in the product $m^{*} \Delta$ [5,9]. Equation (38) provides a good approximation of the exact Bloch energies close to the merging point, as it is demonstrated in Fig. 7 (a similar expansion can be derived around a generic Dirac point). In this figure, we show band cuts along orthogonal directions at $k_{x}=0$ (upper panels) and $k_{y}=$ 1 (lower panels) calculated at $\boldsymbol{k}_{M}=(0,1)$, i.e., the top corner of the first Brillouin zone (see Fig. 6). Panels (a) and (c) show two Dirac points belonging to adjacent Brillouin zones, symmetric with respect to $k_{y}=1$ [see Eq. (28)]. This corresponds to a positive $\Delta$ (in the present regime of parameters $m^{*}$ is always negative). By decreasing $V_{\bar{X}}$ the two Dirac points approach each other and eventually merge when $\Delta=0$, as shown in panels (b) and (e). At this particular point, the dispersion law is linear along $k_{x}$ and quadratic along $k_{y}$. By further decreasing $V_{\bar{X}}$, a gap opens at the merging point, as shown in panels (c) and (f). In this case, the masslike term is characterized by a negative $\Delta$. In all the panels, the bands are compared with the low-energy expansion (38), showing a very good agreement close to the merging point [except panel (d); see the caption of the figure]. In particular, a small asymmetry in the quadratic behavior along $k_{y}$ is visible and well reproduced close to the merging point, owing to the diagonal term proportional to $1 / 2 \mu$ in Eq. (37) [25].
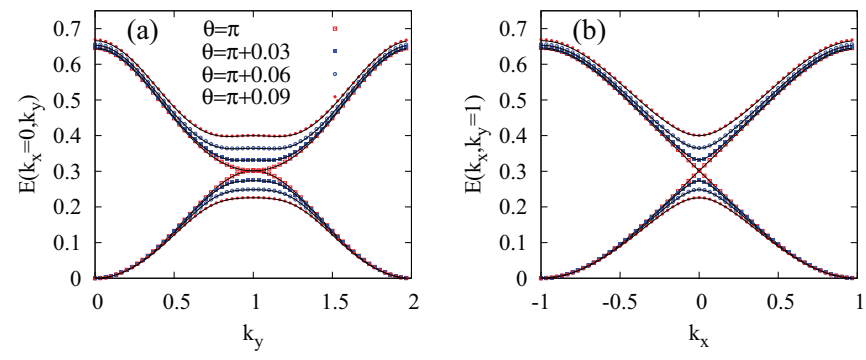

FIG. 8. (Color online) Cuts of the energy bands around the merging point $\boldsymbol{k}_{M}=(0,1)$ for different values of the parity breaking angle $\theta$. The exact Bloch bands (dots) are compared to the full tight-binding model (solid line), as a function of $k_{y}$ (at $k_{x}=0$ ) (a), and of $k_{x}$ (at $\left.k_{y}=1\right)$ (b). The picture refers to the tight-binding regime $V_{X}=0.56, V_{Y}=3.6$, and $V_{\bar{X}}=6.54$. 

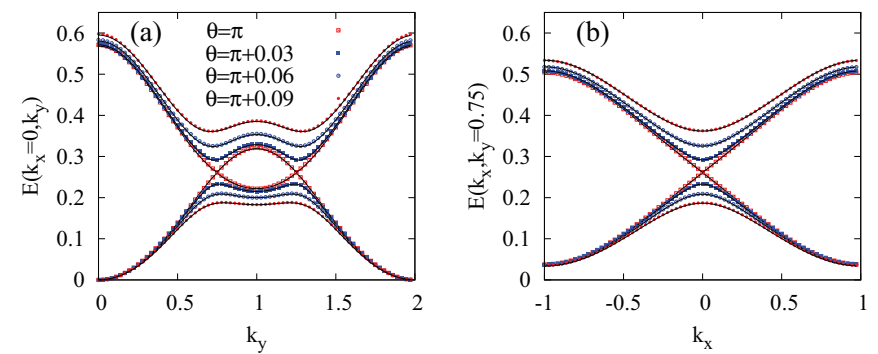

FIG. 9. (Color online) Same as Fig. 8, but for a case with two Dirac points located at $\boldsymbol{k}=(0,0.75)\left(V_{X}=0.56, V_{Y}=3.6\right.$, and $V_{\bar{X}}=$ 8.05).

\section{B. Breaking parity: Massive Dirac points}

As demonstrated experimentally in Ref. [8], a gap can be opened at the Dirac points by breaking the invariance under parity, which is achieved by tuning the angle $\theta$ away from $\pi$. In this case, due to the asymmetry of the two minima in the unit cell, the diagonal terms $\epsilon_{v}$ and $j^{v}(\nu=A, B)$ are no longer degenerate, as shown in Appendix D. As a consequence, the Dirac particles acquire a finite mass, as it is evident from Figs. 8 and 9, where we show the energy bands for two Dirac points, at $\boldsymbol{k}=(0,0.75)$ and at the merging point $\boldsymbol{k}_{M}=(0,1)$. These figures show that even small deviations from $\theta=\pi$ give rise to a significant mass contribution (gap) at the Dirac points, and that the the current tight-binding model accurately reproduces the exact energy bands. We note that in this case the full Eq. (12) has to be used without approximation. We also mention that even in this case one can derive an expansion analog to that in Eq. (38).

\section{CONCLUSIONS}

Maximally localized Wannier functions for composite bands [12] are a powerful tool for constructing tight-binding models for ultracold atoms in optical lattices. Here, we have considered the tunable honeycomb optical lattice of Ref. [8], and we have shown how to derive the corresponding tight-binding Hamiltonian, ab initio. We have calculated the MLWFs and the tunneling coefficients for different lattice configurations, showing that the spectrum properties, including the position of Dirac points and the dispersion law close to their merging, can be reproduced with high accuracy with an expansion up to third-nearest neighbors. We have considered both cases of massless and massive Dirac points, respectively, for the case of two degenerate minima per unit cell and for the case of parity breaking. These results provide a direct connection between the experimental results of Ref. [8] and the universal Hamiltonian of Refs. [5,9].

\section{ACKNOWLEDGMENTS}

This work has been supported by the UPV/EHU under programs UFI 11/55 and IT-366-07, the Spanish Ministry of Science and Innovation through Grants No. FIS2010-19609C02-00 and No. FIS2012-36673-C03-03, and the Basque Government through Grant No. IT-472-10.

\section{APPENDIX A: APPROXIMATE BLOCH SPECTRUM}

In this appendix we briefly review the application of the MLWFs for the tight-binding expansion of the Bloch spectrum, following the discussion of Ref. [12] (see also [13]). Let us start by rewriting the the Hamiltonian density in Eq. (7) [see Eq. (3)],

$$
\begin{aligned}
h_{\nu \nu^{\prime}}(\boldsymbol{k}) & =\sum_{i} e^{i \boldsymbol{k} \cdot \boldsymbol{R}_{i}}\left\langle w_{\mathbf{0} v}\left|\hat{H}_{0}\right| w_{\boldsymbol{i} \nu^{\prime}}\right\rangle \\
& =\frac{1}{V_{B}} \sum_{\boldsymbol{i}} \int_{\mathcal{B}} d \boldsymbol{q} e^{i(\boldsymbol{k}-\boldsymbol{q}) \cdot \boldsymbol{R}_{i}} \sum_{n} U_{\nu n}^{*}(\boldsymbol{q}) U_{\nu^{\prime} n}(\boldsymbol{q}) \varepsilon_{n}(\boldsymbol{q}),
\end{aligned}
$$

where $\varepsilon_{n}(\boldsymbol{k})$ are the (exact) Bloch bands, and the $U_{v n}(\boldsymbol{q})$ unitary matrices are periodic, and represent gauge transformations (as a function of quasimomentum) of the Bloch states.

Single-band case. For the $U(1)$ group transformations that do not mix the bands, namely when

$$
U_{v m}(\boldsymbol{k})=e^{i \phi_{v}(\boldsymbol{k})} \delta_{v m},
$$

the on-site energies and tunneling coefficients $\left\langle w_{\mathbf{0} v}\left|\hat{H}_{0}\right| w_{\boldsymbol{i} v^{\prime}}\right\rangle$ in (A1) are independent on the (periodic) phases $\phi_{\nu}(\boldsymbol{k})$. Furthermore, if all the terms in the sum are retained, owing to the following formula (valid for an infinite lattice):

$$
\frac{1}{V_{B}} \sum_{i} e^{i \boldsymbol{R}_{i} \cdot\left(\boldsymbol{k}^{\prime}-\boldsymbol{k}\right)}=\delta\left(\boldsymbol{k}^{\prime}-\boldsymbol{k}\right),
$$

one easily recovers the exact diagonal expression $h_{\nu \nu^{\prime}}(\boldsymbol{k})=$ $\delta_{v v^{\prime}} \varepsilon_{v}(\boldsymbol{k})$. As a consequence, in the case of a single band, the tunneling coefficients can be expanded in terms of the exact energies (with no reference to the Wannier functions) [26,27]. Moreover, the tight-binding approximation of the exact Bloch spectrum, namely the truncation of the sum in (A1) at a given order, is independent on the choice of the Wannier states. So, in the absence of band mixing [the gauge group being a direct product of $U(1)$ groups], the tight-binding expansion is gauge independent.

Composite-band case. Let us now consider the case of composing $N$ bands, via a non-Abelian $U(N)$ gauge transformation. Again, summing over all lattice sites the Hamiltonian density in Eq. (A1) takes the form

$$
h_{v v^{\prime}}(\boldsymbol{k})=\sum_{n} U_{v n}^{*}(\boldsymbol{k}) U_{v^{\prime} n}(\boldsymbol{k}) \varepsilon_{n}(\boldsymbol{k}),
$$

whose eigenvalues coincide with the exact bands $\varepsilon_{v}(\boldsymbol{k})$ by construction. Moreover, even the trace of $h_{v v^{\prime}}(\boldsymbol{k})$ in Eq. (A1) is gauge independent. Instead, finite order approximations of individual Bloch bands are gauge dependent, as they depend on a particular choice of the matrices $U_{n m}(\boldsymbol{k})$.

Parallel transport gauge. We recall that the transformation matrices $U_{n m}(\boldsymbol{k})$ are obtained by minimizing the Wannier spread $\Omega=\sum_{\nu}\left[\left\langle\boldsymbol{r}^{2}\right\rangle_{\nu}-\langle\boldsymbol{r}\rangle_{\nu}^{2}\right]$, and that the latter can be decomposed as $\Omega=\Omega_{I}+\tilde{\Omega}$ [12]. The first term is gauge invariant, while - in the case of composite bands - the second can be written as the sum of two (non-negative) diagonal and off-diagonal components, $\tilde{\Omega}=\Omega_{D}+\Omega_{O D}$. Both $\Omega_{D}$ and $\Omega_{O D}$ can be written in terms of the generalized Berry vector potentials $\boldsymbol{A}_{v v^{\prime}}(\boldsymbol{k})$, defined as [28,29]

$$
\boldsymbol{A}_{v v^{\prime}}(\boldsymbol{k})=i V_{B}\left\langle u_{v \boldsymbol{k}}\left|\nabla_{\boldsymbol{k}}\right| u_{v^{\prime} \boldsymbol{k}}\right\rangle
$$


with the matrix $\boldsymbol{A}_{v v^{\prime}}(\boldsymbol{k})$ being Hermitian. In one dimension, the gauge in which $\Omega$ is minimized corresponds to $\Omega_{O D}=0$ as a consequence of the vanishing of off-diagonal $\left(v \neq v^{\prime}\right)$ Berry connections in Eq. (A5), which is the so-called parallel transport gauge. In this case, the transformation $U(k)$ can be obtained directly by requiring the off-diagonal connections to be vanishing [29]. Nevertheless, as the Wannier functions are gauge dependent, the two points of view are correlated. We remark that this approach is generally limited to $1 \mathrm{D}$ cases, as in higher dimensions it is not always possible to make $\Omega_{O D}$ vanishing [12], so that the parallel-transport formulation cannot be easily generalized. However, though in absence of a formal proof, in general we may assume that the gauge where the spread of Wannier functions is minimal is the one that provides the best tight-binding approximation of individual Bloch bands. In fact, this has been already verified in a number of models [14-16].

Finally, we remark that the use of composite instead of single-band transformations is required in the case of a set of almost degenerate bands (well separated from the others), that usually corresponds to more that one minimum per unit cell, as is the case of the present work. A more thorough discussion on this point, for the case of a 1D double-well potential, can be found in [14].

\section{APPENDIX B: MLWFS AND TUNNELING COEFFICIENTS}

In this appendix we analyze the properties of the MLWFs and the associated tunneling coefficients in a range of $V_{\bar{X}}$ broader than the one covered in the body of our article. This allows us to analyze the two opposite limits of the potential (8) corresponding to the dimer and 1D chain structures [8]. These are exemplified in Figs. 10(a) and 10(b) $\left(V_{\bar{X}}=1\right.$ and $V_{\bar{X}}=$ 8 , respectively) for the experimental regime of Ref. [8]. The dimer structure is characterized by a relatively low value of the potential in the region between $A$ and $B$ within the same unit cell. On the opposite, in the $1 \mathrm{D}$ chain regime the potential is low along the $y$ direction connecting different minima, while it presents a barrier between the $A$ and $B$ sites of the same unit cell. The stretched-honeycomb regime covered in the text (Fig. 2) represents an intermediate configuration between these two limits.

The structure of the potential in these limits determines the shape of the MLWFs, which we illustrate in Figs. 10(c) and $10(\mathrm{~d})$ (results shown for sublattice $A$ ). As in the stretchedhoneycomb structure, the MLWFs are exponentially localized around the $A$ site of the central unit cell and present a non-negligible contribution around the neighboring potential minima. In the case $V_{\bar{X}}=1$ [see Fig. 10(c)] we find a large contribution of the MLWF around the $B$ site of the central unit cell, consistent with the dimer structure of the potential. The situation is very different for $V_{\bar{X}}=8$ [in Fig. 10(d)], which shows a MLWF highly localized along the $y$ axis, resembling the $1 \mathrm{D}$ chain structure of the potential.

In order to analyze the degree of localization of the MLWFs, in Fig. 11 we show the spread of the MLWFs, $\Omega=\sum_{v=1}^{2}\left[\left\langle\boldsymbol{r}^{2}\right\rangle_{v}-\langle\boldsymbol{r}\rangle_{v}^{2}\right][12]$, as a function of $V_{\bar{X}}$. The figure shows that, by increasing $V_{\bar{X}}, \Omega$ rapidly decreases in the regime of low $V_{\bar{X}}$, while it almost saturates in the opposite limit. This indicates that the tight-binding approach is expected to work
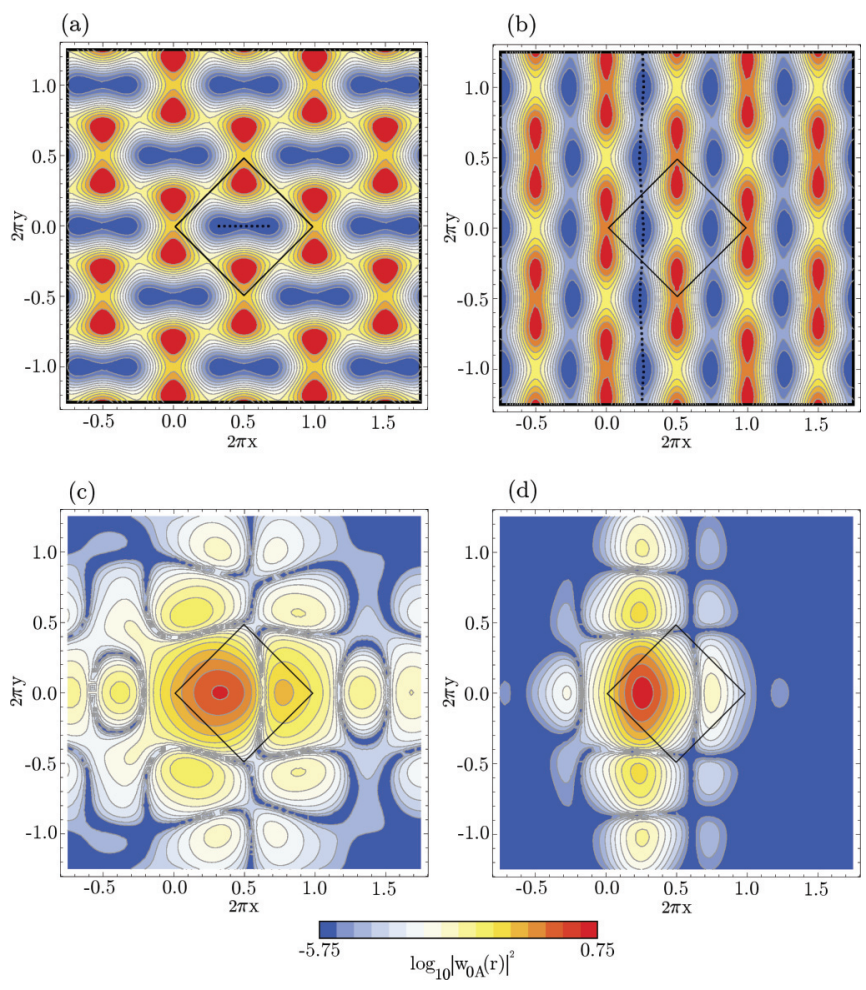

FIG. 10. (Color online) Dimer $\left(V_{\bar{X}}=1\right)$ and 1D-chain $\left(V_{\bar{X}}=8\right)$ limits for fixed $V_{X}=0.28$ and $V_{Y}=1.8$. Panels (a) and (b) show the respective potential structures, and (c) and (d) show associated MLWFs. Color code as in Fig. 2.

better in the stretched-honeycomb and the 1D-chain regimes, rather than for the dimer case.

The behavior of the tunneling coefficients in the whole range from the dimer to the $1 \mathrm{D}$ chain limits are shown in Fig. 12. We first focus on the left-hand side of the graphic, $V_{\bar{X}} \simeq 1 E_{R}$. There, we find that the ratio between the two dominant coefficients is $t_{0} / t_{1} \simeq 10$. This reflects the dimer structure of the potential, since $t_{0}$ connects sites $A$ and $B$ (see Fig. 1). Noteworthy, $t_{2}$ is by far the next biggest coefficient, comparable in magnitude to $t_{1}$. This fact reveals that the tunneling between neighboring dimers in $x$ direction

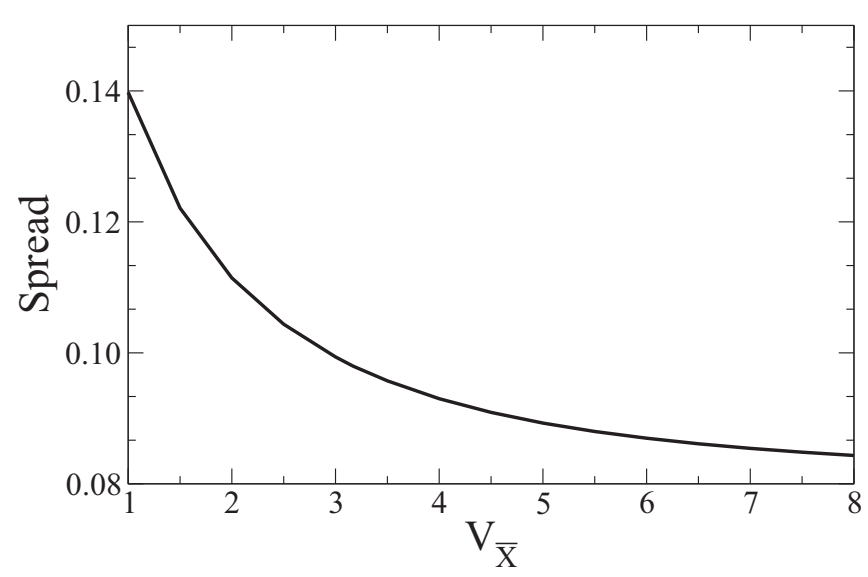

FIG. 11. Spread of the MLWFs as a function of $V_{\bar{X}}$, in the regime of the experiment [8] $\left(V_{X}=0.28\right.$ and $\left.V_{Y}=1.8\right)$. 


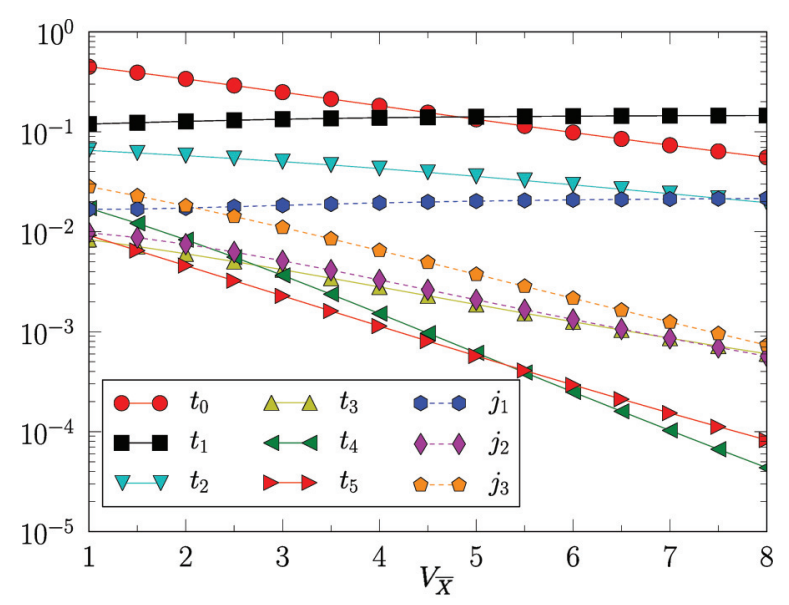

FIG. 12. (Color online) Evolution of various tunneling coefficients as a function of $V_{\bar{X}}$, covering the whole range from the dimer $\left(V_{\bar{X}} \approx 1\right)$ to the $1 \mathrm{D}$-chain $\left(V_{\bar{X}} \approx 1\right)$ limits in the regime of the experiment [8] $\left(V_{X}=0.28\right.$ and $\left.V_{Y}=1.8\right)$.

is considerable (see $t_{2}$ in Fig. 1). The rest of the coefficients have a significantly lower value than those corresponding to $t_{0}, t_{1}$, and $t_{2}$. We note that in Fig. 12 there are two coefficients, $t_{4} \equiv T_{11}$ and $t_{5} \equiv-T_{-10}=-T_{0-1}$ [see Eq. (16)], that were not considered in our original expansion. In the dimer regime, these coefficients can be larger than $j_{1}, j_{2}$, and $t_{3}$, included in our tight-binding model.

As $V_{\bar{X}}$ is increased, the various tunneling coefficients evolve in two different ways. Most of them decrease in magnitude, reflecting the stronger localization of the MLWFs as we approach a more tight-binding regime. This could be termed as the "normal" behavior, which is followed for instance by the tunneling coefficients of a perfect honeycomb lattice [15,24]. However, two coefficients, namely $t_{1}$ and $j_{1}$, increase in magnitude as $V_{\bar{X}}$ is increased. This apparently "inverse" behavior reflects the evolution of the potential in Eq. (8) from the dimer to the 1D chain structure, as these coefficients connect potential minima inside the 1D chains. Owing to this inverse behavior, $t_{1}$ becomes the dominant coefficient for $V_{\bar{X}} \gtrsim 4.5 E_{R}$. Similarly, $j_{1}$ becomes larger than $j_{3}$ and even $t_{2}$ for $V_{\bar{X}} \gtrsim 7.5$. Thus it is clear that varying the potential amplitude can modify the role of the different tunneling coefficients.

\section{APPENDIX C: ACCURACY OF THE TIGHT-BINDING MODELS}

In Figs. 13(a) and 13(b) we compare the exact and tightbinding energy dispersions along $k_{y}\left(k_{x}=0\right)$ in the dimer and 1D-chain limits [panels (a) and (b), respectively], for the experimental regime [8]. Here we have included the results for the two tight-binding approximations considered in the text (with just $t_{0}, t_{1}, t_{2}$, and with all the coefficients). As in the stretched-honeycomb case (Fig. 4), the tight-binding model reproduces the main features of the exact dispersion, including the approximate position of the Dirac point in the case of Fig. 13(b) [note that there is no such point in the dimer limit, Fig. 13(a)]. In Figs. 13(c) and 13(d) we show the analogous pictures for the tight-binding regime discussed in the text, that is doubling the potential parameters of Ref. [8]. In this case,
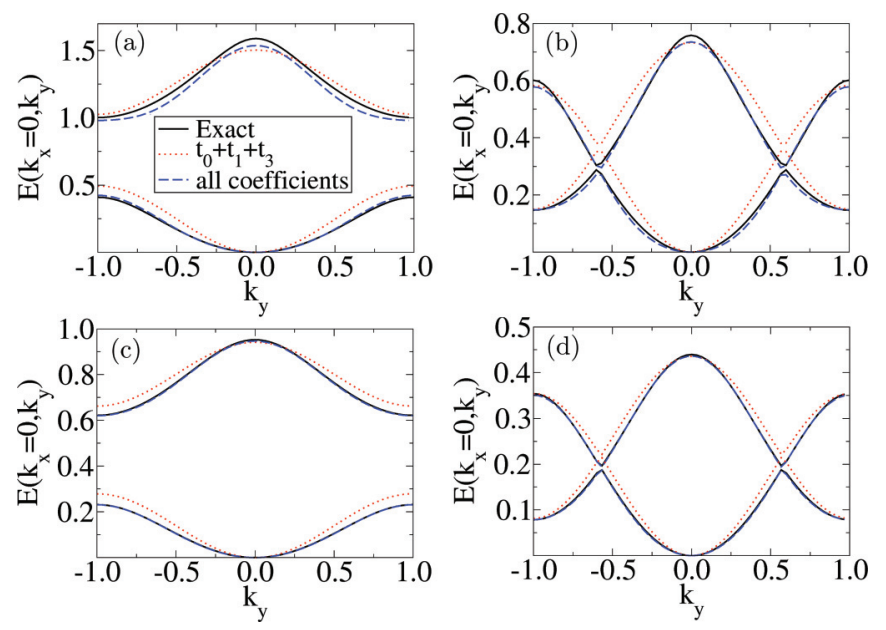

FIG. 13. (Color online) Cut of the exact energy bands along $k_{y}$ $\left(k_{x}=0\right)$ compared to the two tight-binding approximations discussed in the text. Panels (a) and (c) represent the dimer limits in the experimental and tight-binding regimes, respectively. Panels (b) and (d) are analogous in the 1D-chain limit.

the agreement with the exact energies when all the coefficients are included is remarkable in both limits.

Another way to test the accuracy of the different tightbinding expansions is to analyze the overall mismatch of the tight-binding energies against the exact ones. Here, we evaluate this mismatch using the following expression:

$$
\delta E_{1,2} \equiv \frac{1}{\bar{\Delta}} \sqrt{\frac{1}{S_{B}} \int_{\mathcal{B}} d \boldsymbol{k}\left[\varepsilon_{1,2}(k)-\epsilon_{-,+}(k)\right]^{2}},
$$

where $\varepsilon_{n}$ are the exact energies, $\bar{\Delta} \equiv\left(\Delta \varepsilon_{1}+\Delta \varepsilon_{2}\right) / 2$ the average bandwidth, and $S_{B}$ the area of the Brillouin zone $[14,15]$. The calculated mismatch $\delta E_{n}$ is shown in Figs. 14(a) and $14(\mathrm{~b})$ as a function of $V_{\bar{X}}$, for the experimental and tightbinding regimes, respectively. Overall, in the tight-binding regime (b), the mismatch is one order of magnitude smaller than the one of the experimental regime (a). Remarkably, the best approximation in Fig. 14(b) has an error below 1\% in all the considered range of parameters. From Fig. 14 we identify two different trends in the behavior of the mismatch. Focusing on Fig. 14(a), we find that, for $V_{\bar{X}} \lesssim 4.5 E_{R}, \delta E_{n}$ decreases as $V_{\bar{X}}$ is increased. This behavior could be expected, since in this parameter range the MLWFs become much more

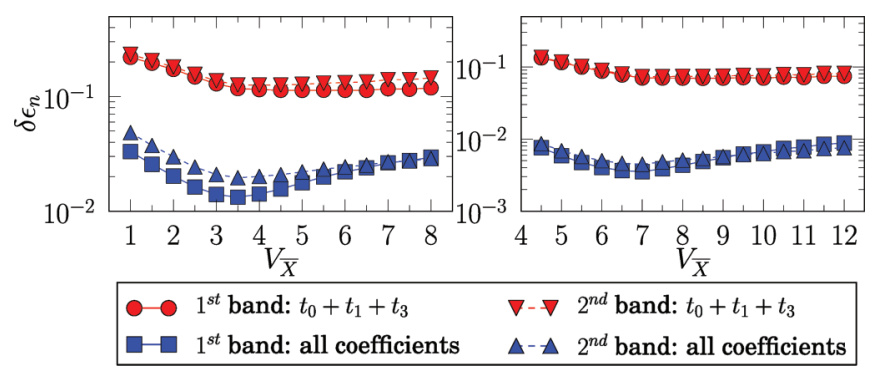

FIG. 14. (Color online) Calculated energy mismatch $\delta E_{n}$ for the two bands including the two tight-binding approximations discussed in the text. Panels (a) and (b) respectively show the results for the experimental and tight-binding regimes. 

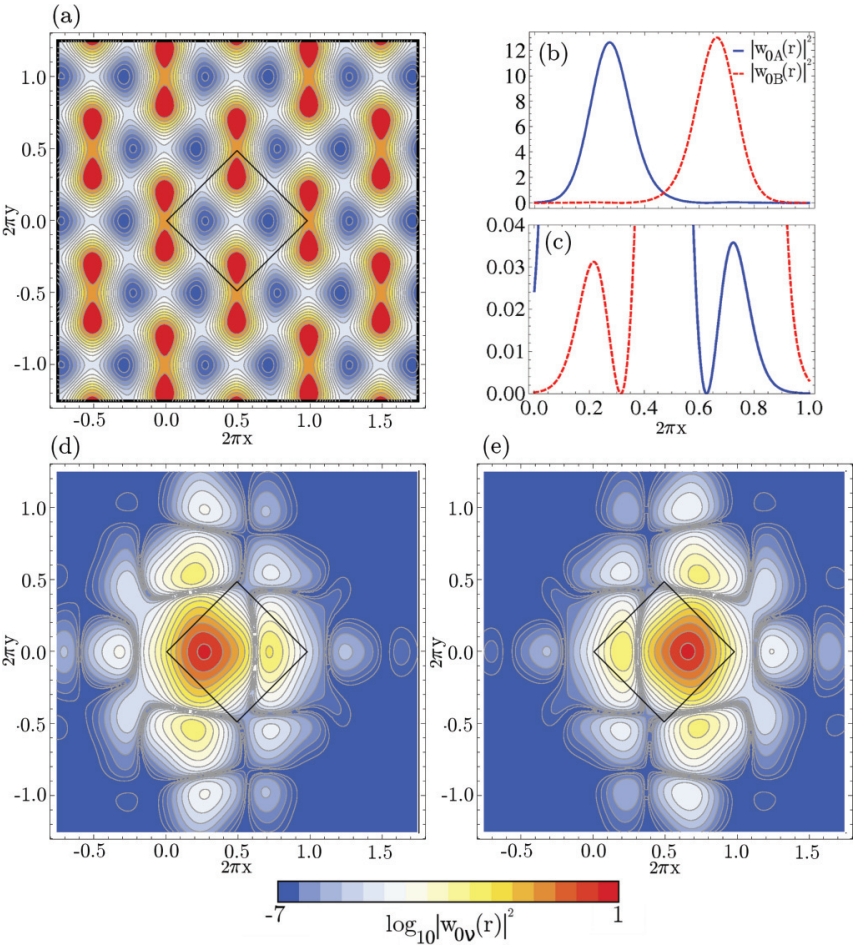

FIG. 15. (Color online) Asymmetric structure corresponding to the angle $\theta=\pi+0.1$, with potential parameters $V_{X}=0.56, V_{Y}=$ 3.6, and $V_{\bar{X}}=6.94$ (merging point). Panel (a) illustrates the structure of the potential for this configuration, showing a deeper minimum at sublattice $B$ than in $A$ (color code as in Fig. 2). Panels (b) and (c) show one-dimensional profiles of $\left|w_{0 A}(x, y=0)\right|^{2}$ (solid, blue) and $\left|w_{0 B}(x, y=0)\right|^{2}$ (dashed, red) in the central unit cell. Note the different distributions of the two MLWFs, as a consequence of parity breaking. This is evident also from the two-dimensional plots of $\left|w_{0 A}(\mathbf{r})\right|^{2}$ and $\left|w_{0 B}(\mathbf{r})\right|^{2}$, in (d) and (e), respectively.

localized as the potential is raised (see Fig. 11); hence a more tight-binding regime is approached. For $V_{\bar{X}} \gtrsim 4.5 E_{R}$, in contrast, the mismatch increases with increasing $V_{\bar{X}}$. We recall from Fig. 12 that the tunneling coefficients corresponding to sites inside the $1 \mathrm{D}$ chains grow as $V_{\bar{X}}$ is increased. When approaching the 1D-chain limit, some of these coefficients

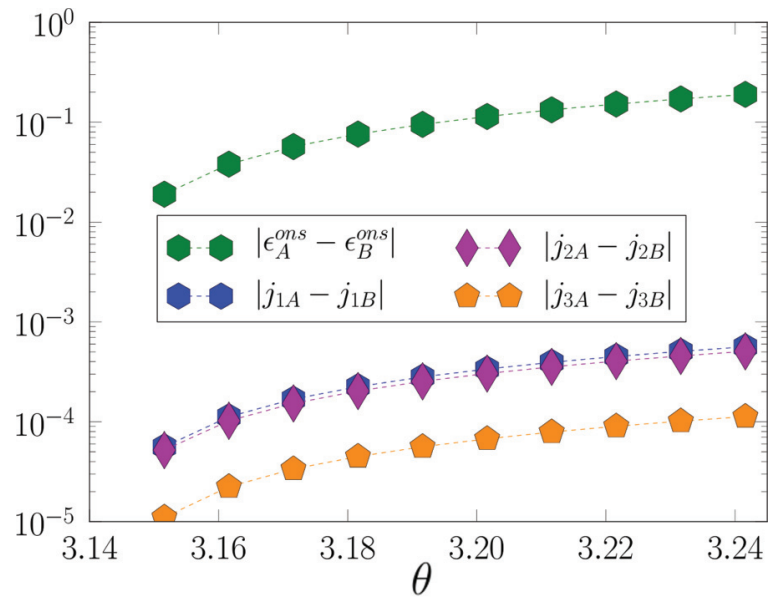

FIG. 16. (Color online) Splitting of the diagonal coefficients as a function of the angle $\theta$ (at the merging point: $V_{X}=0.56, V_{Y}=3.6$, and $V_{\bar{X}}=6.94$; cf. Fig. 3). Note that $\left|E_{A}-E_{B}\right|=2|\epsilon|$; see Eq. (19).

that are not considered in our tight-binding model may become relevant; hence the quality of the approximation may decrease.

\section{APPENDIX D: EFFECT OF PARITY BREAKING}

Here we analyze the asymmetric case corresponding to $\theta \neq$ $\pi$, considering for simplicity just the tight-binding parameter regime. In this configuration, the two potential minima in the unit cell become nondegenerate [8]. In Fig. 15(a) we illustrate the structure of the potential for $\theta=\pi+0.1$ at the merging point, with the deeper minimum at site $B$. Correspondingly, the associated MLWFs exhibit a higher localization around $B$, as illustrated in Figs. 15(b)-15(e). As a consequence of parity breaking, the degeneracy of the diagonal coefficients is also broken [for both the on-site energies $E_{v}=J_{00}^{\nu}$-see Eqs. (17)-(19)—and the tunneling coefficients $\left.j_{i}^{v}, v=A, B\right]$; see Fig. 16. This figure shows the splitting of on-site energies and diagonal tunneling coefficients for small deviations from $\theta=\pi$ (the off-diagonal tunneling coefficients are weakly affected in this range of values of $\theta$ ). These variations allow one to accurately reproduce the exact dispersion law and in particular the opening of a mass gap at the Dirac points, as discussed in the text (see Figs. 8 and 9).
[1] S.-L. Zhu, B. Wang, and L.-M. Duan, Phys. Rev. Lett. 98, 260402 (2007).

[2] C. Wu, D. Bergman, L. Balents, and S. Das Sarma, Phys. Rev. Lett. 99, 070401 (2007); C. Wu and S. Das Sarma, Phys. Rev. B 77, 235107 (2008).

[3] B. Wunsch, F. Guinea, and F. Sols, New J. Phys. 10, 103027 (2008).

[4] K. L. Lee, B. Grémaud, R. Han, B.-G. Englert, and C. Miniatura, Phys. Rev. A 80, 043411 (2009).

[5] G. Montambaux, F. Piéchon, J. N. Fuchs, and M. O. Goerbig, Phys. Rev. B 80, 153412 (2009); Eur. Phys. J. B 72, 509 (2009); J. N. Fuchs, L. K. Lim, and G. Montambaux, Phys. Rev. A 86, 063613 (2012); R. de Gail, J. N. Fuchs, M. O.
Goerbig, F. Piéchon, and G. Montambaux, Physica B 407, 1948 (2012).

[6] P. Soltan-Panahi, J. Struck, P. Hauke, A. Bick, W. Plenkers, G. Meineke, C. Becker, P. Windpassinger, M. Lewenstein, and K. Sengstock, Nature Phys. 7, 434 (2011); P. Soltan-Panahi, D.-S. Lühmann, J. Struck, P. Windpassinger, and K. Sengstock, ibid. 8, 71 (2011).

[7] K. Sun, W. V. Liu, A. Hemmerich, and S. Das Sarma, Nature Phys. 8, 67 (2012).

[8] L. Tarruell, D. Greif, T. Uehlinger, G. Jotzu, and T. Esslinger, Nature (London) 483, 302 (2012).

[9] L.-K. Lim, J.-N. Fuchs, and G. Montambaux, Phys. Rev. Lett. 108, 175303 (2012). 
[10] Y. Hasegawa and K. Kishigi, Phys. Rev. B 86, 165430 (2012).

[11] T. Uehlinger, D. Greif, G. Jotzu, L. Tarruell, T. Esslinger, L. Wang, and M. Troyer, Eur. Phys. J.: Spec. Top. 217, 121 (2013).

[12] N. Marzari and D. Vanderbilt, Phys. Rev. B 56, 12847 (1997).

[13] N. Marzari, A. A. Mostofi, J. R. Yates, I. Souza, and D. Vanderbilt, Rev. Mod. Phys. 84, 1419 (2012).

[14] M. Modugno and G. Pettini, New J. Phys. 14, 055004 (2012).

[15] J. Ibañez-Azpiroz, A. Eiguren, A. Bergara, G. Pettini, and M. Modugno, Phys. Rev. A 87, 011602(R) (2013).

[16] R. Walters, G. Cotugno, T. H. Johnson, S. R. Clark, and D. Jaksch, Phys. Rev. A 87, 043613 (2013).

[17] D. Jaksch, C. Bruder, J. I. Cirac, C. W. Gardiner, and P. Zoller, Phys. Rev. Lett. 81, 3108 (1998).

[18] C. Brouder, G. Panati, M. Calandra, C. Mourougane, and N. Marzari, Phys. Rev. Lett. 98, 046402 (2007).

[19] G. Panati and A. Pisante, Commun. Math. Phys. 322, 835 (2013).
[20] B. Vaucher, S. R. Clark, U. Dorner, and D. Jaksch, New J. Phys. 9, 221 (2007).

[21] A. Mostofi, J. Yates, Y. Lee, I. Souza, D. Vanderbilt, and N. Marzari, Comput. Phys. Commun. 178, 685 (2008).

[22] P. Giannozzi et al., J. Phys. Condens. Matter 21, 395502 (2009).

[23] The correspondence with the tunneling coefficients of Ref. [9] is the following: $t_{0} \rightarrow t^{\prime}, t_{1} \rightarrow t$, and $t_{2} \rightarrow t^{\prime \prime}$ (notice that in principle $t^{\prime}$ and $t^{\prime \prime}$ can be interchanged).

[24] We notice that in the case of a perfect honeycomb geometry one has $t_{1}=t_{0}, t_{2}=t_{3}$, and $j_{1}^{v}=j_{2}^{v}$, whereas $j_{3}^{v}$ can be safely disregarded in the tight-binding regime; see [14].

[25] This term is not considered in the universal model of Refs. [5,9].

[26] L. He and D. Vanderbilt, Phys. Rev. Lett. 86, 5341 (2001).

[27] G. L. Alfimov, P. G. Kevrekidis, V. V. Konotop, and M. Salerno, Phys. Rev. E 66, 046608 (2002).

[28] M. C. Chang and Q. Niu, Phys. Rev. Lett. 75, 1348 (1995); Phys. Rev. B 53, 7010 (1996).

[29] G. Pettini and M. Modugno, Phys. Rev. A 83, 013619 (2011). 